\title{
Galactooligosaccharides and substrate and energy metabolism, dietary intake and body composition
}

\author{
M.A.J. van Dijck \\ Maastricht University \\ maj.vandijck@student.maastrichtuniversity.nl
}

\section{Abstract}

Aim: Obesity is associated with an altered composition of the gut microbiota. Prebiotics such as galactooligosaccharides (GOS) might modulate the gut microbiota composition beneficially and might have favorable metabolic effects in overweight and obese subjects. The aim of the study is to determine the effect of GOS on human substrate and energy metabolism, dietary intake and body composition in overweight and obese subjects. Methods: In a placebo-controlled, double-blind, randomized, parallel study, the effects of GOS were investigated in overweight and obese subjects. Weight-stable men and post-menopausal women between 40 and 75 years were included. Before and after the 12-week intervention period human substrate and energy expenditure were measured by indirect calorimetry. Subjects reported their dietary intake before, in week 6 and after the intervention period by completing a three-day weighed food diary. Self-reported level of physical activity was assessed using the validated SOUASH questionnaire. Changes in body composition were measured using a Dual-energy X-ray absorptiometry (DEXA) scan. A two-factor mixed analysis of variance (ANOVA) was performed to compare the intervention and placebo group. All analyses were performed blinded. Results: This interim analysis was performed after the data of 25 subjects was available. The data was blinded while analyzing. To maintain confidentiality of the data, the results of this interim analysis were not published. Conclusion: Only a part of the whole subject population was included in this interim analysis. The analyses in the total study group are required to draw more definite conclusions on the metabolic effects of GOS and to outline the potential of GOS to favorably affect host metabolism of overweight and obese subjects.

\section{Keywords}

Prebiotics, Galactooligosaccharides, Obesity, Energy metabolism, Dietary intake, Body composition. 


\section{Introduction}

The prevalence of obesity has increased dramatically. In the Netherlands, the prevalence of obesity increased from 5\% to 12\% between 1981 and 2012 (1). The increased availability of high-fat and sugar products and decreased physical activity in the modern Western society result in accumulation of excess body fat in individuals (2). Recent studies support the idea of the involvement of the gut microbiota in host metabolism and metabolic diseases such as obesity (3). In obesity the bacterial diversity of the gut microbiota is reduced and the composition of the gut microbiota is altered (4). In contrast to the human genome, the collective genome of the micro-organisms in the gastro-intestinal tract, the microbiome, can be modulated. Prebiotics are non-digestible carbohydrates that can modulate the composition of the gut microbiota and thereby improving host health (5). Gut microbiota ferment prebiotics into short chain fatty acids (SCFA) in the colon. The role of SCFA in energy homeostasis is controversial. SCFA production might lead to increased energy harvest, thereby putatively contributing to obesity and related disorders, but in contrast several animal and human studies showed beneficial metabolic effects of SCFA (6). Several animal studies found a decrease in fat mass together with a decrease in dietary intake after feeding with prebiotics (7). Increased levels of Glucagonlike peptide (GLP)-1 and peptide tyrosine tyrosine (PYY) were found after feeding rats and mice with prebiotics (7). Furthermore, recent animal studies have described the role of SCFA in increased energy expenditure and fat oxidation (8). Therefore, the production of SCFA might have anti-obesity effects in humans.

Galactooligosaccharide (GOS) belongs to a group of soluble fibers of which the prebiotic effect has been shown in clinical trials (7). However, current knowledge about the metabolic effects of GOS administration in humans is limited. Recently, Vulevic et al. (9) studied the effect of GOS on markers of the metabolic syndrome in overweight adults. Beneficial metabolic effects as reduced fasting insulin concentrations and improved lipid profiles were found following 12-week administration of GOS. These beneficial effects may be related to an increase in Bifidobacteria (9). Little information is known about the fermentation of GOS into SCFA. SCFA are end products of bacteria having a saccharolytic metabolism, such as Bifidobacteria and Lactobacilli (10). GOS has been shown to be bifidogenic (9) and thus could increase the production of SCFA. Based on the hypothesis that administration of GOS increases the production of SCFA, GOS might have beneficial effects on human substrate and energy metabolism. Furthermore, increased production of SCFA might reduce dietary intake. Increased energy expenditure and fat oxidation and decreased energy intake could improve the energy balance, resulting in improved 
body weight control. The aim of the study is to determine the effect of GOS on substrate and energy metabolism, dietary intake and body composition in overweight and obese subjects.

\section{Material and methods}

The effects of GOS were investigated in a placebo-controlled, double-blind, randomized, parallel study. The Medical Ethical Committee of Maastricht University and academic hospital provided ethical approval for the study.

\section{Subjects}

Overweight or obese (body mass index (BMI) $\geq 28 \mathrm{~kg} / \mathrm{m} 2<40 \mathrm{~kg} / \mathrm{m} 2$ ) men and postmenopausal women between 40 and 75 years were recruited in the vicinity of Maastricht. Volunteers had to be weight-stable for at least 3 months prior to participation in the study. During a screening visit, glucose tolerance of the volunteers was determined by an Oral Glucose Tolerance Test (OGTT).Volunteers with impaired glucose tolerance (plasma glucose of 7.8-11.1 mmol// after 2 hours OGTT) and/or impaired fasting glucose (plasma glucose ffl $5.6 \mathrm{mmol} / \mathrm{l}$ in fasted state) were included. Exclusion criteria were use of supplements of pre- or probiotic products regularly (>3 times per week) and use of antibiotics in the last three months or during the study period.

\section{Experimental design}

Subjects were randomly assigned to the GOS or placebo group using block randomization to match the subjects for gender, age and BMI. Both the subjects and the researchers were blinded. Subjects in the intervention group were asked to ingest $7.04 \mathrm{~g}$ of Domo ${ }^{\circledR}$ Vivinal $^{\circledR}$ galactooligosaccharide rich whey product (which contains 5 g GOS) three times per day during the 12-week intervention period. Subjects in the placebo group were asked to ingest $5.65 \mathrm{~g}$ of Maltodextrin three times per day during 12 weeks. The amount of Maltodextrin ( $211 \mathrm{~kJ} /$ day) was isocaloric to the amount of GOS (210 kJ/day). The products were consumed with a low fat yogurt drink (Optimel langlekker 200ml, Friesland Campina) during breakfast, lunch and dinner. All subjects were advised to continue their usual exercise and dietary regimen throughout the intervention period.

\section{Study parameters}

Approximately one week after the screening visit, body composition and substrate and energy metabolism were measured. During the days prior to this test day subjects recorded their dietary intake and level of physical activity. The 12-week intervention period started 
on the day after the test day. In week six of the intervention period, subjects recorded again their dietary intake and level of physical activity. After the 12-week intervention period the measurements of the same study parameters as before the intervention were repeated.

\section{Dietary intake}

Subjects were asked to complete a three-day weighed dietary record before the intervention period, in week 6 and after the intervention period. Before the start of the intervention subjects were instructed how to weigh and record their food and beverage intake. The subjects were asked to include two week days and one weekend day in the three-day period. The dietary records were analyzed with the use of Eetmeter software (Voedingscentrum, The Hague, The Netherlands). Outcome variables were total energy intake and energy intake from carbohydrates, fats, saturated fats and proteins.

\section{Body composition}

Body composition was measured before and after the intervention period by use of a DEXA scan. The subject was lying in a supine position while the whole body was scanned. The effective radiation dose which the subjects received during the scans was approximately 20 microSievert and can be neglected. The body fat percentage, body fat distribution and lean mass of the subjects were quantified by the DEXA scan.

\section{Substrate and energy metabolism}

Resting energy expenditure, fat and carbohydrate oxidation were determined by indirect calorimetry using an open-circuit ventilated hood system (Omnical, Maastricht University, The Netherlands). The subjects received a standardized evening meal on the day before the measurement. After an overnight fast, oxygen and carbon dioxide concentrations in inspired and expired air were measured continuously for 30 minutes. A transparent ventilated hood was placed over the subject's head, while the subject was in a semirecumbent position. Afterwards, a one-step hyperinsulinemiceuglycemic clamp was performed to measure insulin sensitivity. During the clamp, glucose and insulin were infused in an antecubital vein of the forearm. The plasma insulin concentration was raised to $100 \mathrm{mU} / \mathrm{L}$ and a variable amount of glucose was infused to maintain euglycaemia (4.8-5.2 mmol/l). The blood glucose concentration reached at a maximum of 90 minutes was kept stable during the final 30 minutes of the clamp. During this steady state of the clamp, ventilated hood measurements were performed again for 30 minutes, reflecting the insulin-stimulated state. Fasting and postprandial ventilated hood measurements were performed before and after the 12-week intervention period. Resting metabolic rate 
and carbohydrate and fat oxidation were calculated using the equations of Weir (11). In addition, the change in respiratory quotient (RO) from the fasted state to the insulinstimulated condition, called metabolic flexibility, was calculated.

\section{Physical activity}

Self-reported level of physical activity was assessed using the validated Short Questionnaire to Asses Health enhancing physical activity (SOUASH) questionnaire. Subjects completed the questionnaire before the intervention period, in week six of the intervention period and after the intervention period. Outcome values were number of minutes per week spend in light, moderate, and vigorous physical activity. Light physical activity was defined as activities with a metabolic equivalent (MET) <4 (40-55y) and <3 $(55+y)$. Moderate physical activity was defined as 4-6.5 MET (40-55y) and 3-5 MET ( $55+y)$. Vigorous physical activity was defined as $>6.5$ MET (40-55y) and $>5(55+y)$. In addition, the questionnaire was used to assess whether subjects met the Dutch guideline for physical activity (Nederlandse Norm Gezond Bewegen (NNGB)): 30 minutes of at least moderate intense physical activity on at least five days of the week.

\section{Statistical analysis}

All statistical tests were performed using SPSS version 21. Descriptive analysis was carried out for all variables. A (two-factor) repeated-measures analysis of variance (ANOVA) was performed to compare the intervention and placebo group for the different outcomes, followed by post hoc tests in case of significance ( $P<0.05)$. Analysis of covariance (ANCOVA) was used to adjust for baseline outcome values, such as age and gender. All reported $p$-values were based on two-sided tests. All analyses were performed blinded.

\section{Results}

This interim analysis was performed after the data of 25 subjects was available. To maintain confidentiality of the data, the results of the interim analysis were not published.

\section{Discussion/Conclusion}

The aim of the study was to determine the effect of GOS on substrate and energy metabolism, dietary intake and body composition in overweight and obese subjects. Since GOS may increase colonic SCFA production through fermentation, we hypothesized that administration of GOS would increase energy expenditure and fat oxidation, decrease dietary intake and improve body composition. In a placebo-controlled, double-blind, randomized, parallel study, the effects of 12-week GOS supplementation were investigated in overweight and obese subjects. 
Animal and human studies have shown that administration of other prebiotic fibers, such as inulin-type fructans, can reduce dietary energy intake (7). Vulevic et al. (9) found no effect of GOS on energy and nutrient intake in overweight adults after 12 weeks of supplementation. However, Overduin et al. (12) found reduced energy intake after feeding young adult male rats with Vivinal ${ }^{\circledR}$ GOS. In their study GOS increased colonic gene expression of PYY and pre-meal and meal-induced plasma levels of PYY. In addition, gene expression of GLP-1 precursor proglucagon was increased. Reduced dietary intake associated with prebiotics might be related to activation of G-protein coupled receptor 41 (GPR41), also called free fatty acid receptor 3 (FFAR3), by SCFA stimulating the secretion of PYY. In addition, SCFA can activate GPR43, also known as FFAR2, thereby promoting the secretion of GLP-1 (6). Therefore, it would be interesting to assess whether GOS supplementation increased PYY and GLP-1 concentrations. In addition, plasma levels of GI peptides will provide more objective information about the effects of GOS on the regulation of dietary intake than self-reported dietary intake, in which energy intake may be underreported.

The intake of dietary fiber has been inversely associated with weight gain (13). In addition, feeding animals with prebiotics resulted in decreased body weight and fat mass (7). In the rodent study of Overduin et al. (12) epididymal fat-pad weight, representing abdominal fat mass, decreased in rats fed with Vivinal ${ }^{\circledR} \mathrm{GOS}$. Reduced dietary intake could be an important mechanism behind the improvements in body weight associated with prebiotics (7).

No animal or human studies are available about the effects of GOS on substrate and energy metabolism. However, it has been suggested that acute infusion of acetate in the distal colon stimulates fat oxidation in overweight males (14). Therefore, it would be interesting to assess whether GOS increased acetate concentrations and to further study the effects of GOS on human substrate and energy metabolism. More double-blind, randomized, placebo-controlled trials are necessary to study the long-term effect of GOS on substrate and energy metabolism in overweight and obese adults. Animal studies suggest that SCFA might promote energy expenditure and fat oxidation (8). However, knowledge is lacking about the effects of SCFA on energy expenditure and fat oxidation in humans. In addition, no studies were available about the effect of GOS on metabolic flexibility. Metabolic flexibility, defined as the change in RO from fasting to insulinmediated conditions, plays an important role in the development of insulin resistance and type 2 diabetes (15). Corpeleijn et al. (15) showed that impaired metabolic flexibility is partly reversible by lifestyle interventions. Therefore, improvements in metabolic flexibility may be beneficial in the prevention of type 2 diabetes. 
This interim analysis may have been insufficiently powered to study differences in dietary intake, body composition and substrate and energy metabolism. Since this study is part of an ongoing study investigating the effect of GOS supplementation on peripheral insulin sensitivity and body weight control, the data of only 25 subjects was available. The study was powered to a minimum of 36 subjects. Therefore, the power may not have been sufficient in this interim analysis and more data have to be available to draw more definite conclusions about the effect of GOS on substrate and energy metabolism, dietary intake and body composition. Moreover, the 12-week intervention period might have been insufficiently long. Vulevic et al. (9) described that modulation of the gut microbiota with GOS supplementation might take longer in overweight subjects. In their study the effect of GOS on the composition of gut microbiota in overweight adults was relatively small compared to other studies in younger and older adults. Furthermore, it is important to reflect on the mixture of GOS used in the study. Mixtures of GOS can differ in purity and the linkages of the oligosaccharide chains can be different due to the use of different enzymes in the production (16). The bifidogenic effect of GOS can be determined by the composition of the GOS mixture.

The present study did not determine the effect of GOS on gut microbiota composition. However, fecal samples before the intervention period, after one week of supplementation and after the intervention period were taken in the ongoing study. Thus, fecal samples will still be analyzed to assess whether GOS supplementation modulated the composition of the gut microbiota. Furthermore, data on fecal and plasma SCFA concentrations was not available in the present study. Besides fecal samples, blood samples were collected in the ongoing study before, after one week of supplementation and after the intervention period. Future research should assess whether 12-week supplementation of GOS was associated with altered SCFA production.

The current knowledge about the effect of GOS in overweight and obese subjects is limited. Therefore, this interim analysis and the ongoing study could provide important insights. These insights are necessary to evaluate the potential of prebiotics such as GOS to prevent and treat obesity and related metabolic disorders. Novel prevention and treatment options for obesity are required, since obesity is considered as a global epidemic (2). Further research is necessary to elucidate the underlying mechanism of the gut microbiota in obesity.

Since only a part of the whole subject population was included in this interim analysis, no final conclusions can be drawn. Furthermore, analyses of SCFA concentrations and GI 
peptides concentrations in blood samples together with analyses of SCFA concentrations and gut microbiota composition in fecal samples will provide more information to elucidate the results so far. The analyses in the total study group are required to draw more definite conclusions on the metabolic effects of GOS and to outline the potential of GOS to favorably affect host metabolism of overweight and obese subjects.

\section{Role of the student}

Miranda van Dijck was an undergraduate student working under the supervision of PhD Student Emanuel Canfora and Prof. dr. Ellen Blaak when the research in this report was performed. Miranda performed practical work during the test days. In addition, she analyzed the results and wrote the report.

\section{References}

1. Bakel A, Zantinge E. Neemt het aantal mensen met overgewicht toe of af. Bilthoven: 2013

2. Kopelman PG. Obesity as a medical problem. Nature. 2000;404(6778):635-43

3. Cani PD, Delzenne NM. Gut microflora as a target for energy and metabolic homeostasis. Curr Opin Clin Nutr Metab Care. 2007;10(6):729-34.

4. Turnbaugh PJ, Hamady M, Yatsunenko T, Cantarel BL, Duncan A, Ley RE, et al. A core gut microbiome in obese and lean twins. Nature. 2009;457(7228):480-4

5. Gibson GR, Roberfroid MB. Dietary modulation of the human colonic microbiota: introducing the concept of prebiotics. J Nutr. 1995;125(6):1401-12.

6. Kasubuchi M, Hasegawa S, Hiramatsu T, Ichimura A, Kimura I. Dietary Gut Microbial Metabolites, Shortchain Fatty Acids, and Host Metabolic Regulation. Nutrients. 2015;7(4):2839-49.

7. Roberfroid M, Gibson GR, Hoyles L, McCartney AL, Rastall R, Rowland I, et al. Prebiotic effects: metabolic and health benefits. Br J Nutr. 2010;104 Suppl 2:S1-63.

8. den Besten G, van Eunen K, Groen AK, Venema K, Reijngoud DJ, Bakker BM. The role of short-chain fatty acids in the interplay between diet, gut microbiota, and host energy metabolism. J Lipid Res 2013;54(9):2325-40.

9. Vulevic J, Juric A, Tzortzis G, Gibson GR. A mixture of trans-galactooligosaccharides reduces markers of metabolic syndrome and modulates the fecal microbiota and immune function of overweight adults. J Nutr. 2013;143(3):324-31.

10. Gibson GR, Scott KP, Rastall RA, Tuohy KM, Hotchkiss A, Dubert-Ferrandon A, et al. Dietary prebiotics: current status and new definition. Food Sci Technol Bull Funct Foods. 2010(7):1-19.

11. Weir JBV. New methods for calculating metabolic rate with special reference to protein metabolism. J Physiol. 1949;109(1-2):1-9. 
12. Overduin J, Schoterman MH, Calame W, Schonewille AJ, Ten Bruggencate SJ. Dietary galactooligosaccharides and calcium: effects on energy intake, fat-pad weight and satiety-related, gastrointestinal hormones in rats. Br J Nutr. 2013;109(7):1338-48.

13. Delzenne NM, Cani PD. A place for dietary fibre in the management of the metabolic syndrome. Curr Opin Clin Nutr Metab Care. 2005;8(6):636-40.

14. van der Beek C, Canfora E, Lenaerts K, Troost F, Holst J, Masclee A, et al. OPo38: Colonic Acetate Infusions Promote Fat Oxidation and Improve Metabolic Parameters in Overweight Males. Clinical Nutrition. 2014(33):S16-S7.

15. Corpeleijn E, Saris WH, Blaak EE. Metabolic flexibility in the development of insulin resistance and type 2 diabetes: effects of lifestyle. Obes Rev. 2009;10(2):178-93.

16. Torres DP, Gonçalves MdPF, Teixeira JA, Rodrigues LR. Galacto-Oligosaccharides: Production, Properties, Applications, and Significance as Prebiotics. Compr Rev Food Sci Food Saf. 2010;9(5):438-54. 